\title{
Domination in Some Operations on Interval-valued Fuzzy Graphs
}

\author{
Ahmed N. Shain \\ Department of Mathematics \\ Faculty of Education and Sciences \\ Sheba Region University, Marib, Yemen \\ Saqr H. Al-Emrany \\ Department of Mathematics \\ Faculty of Education and Sciences \\ Sheba Region University, Marib, Yemen
}

\author{
Mahiuob M.Q. Shubatah \\ Department of Mathematics \\ Faculty of Sciences and Education \\ AL-Baydha University, AL-Baydha, Yemen \\ Nojood A. Al-Khadari \\ Department of Mathematics \\ Faculty of Education and Sciences \\ Sheba Region University, Marib, Yemen
}

\begin{abstract}
In this paper, the domination number $\gamma$ of some operations on interval-valued fuzzy graphs was introduced and established investigated for some operations on bipolar fuzzy graphs such as union, join, Cartesian product, strong product and composition.
\end{abstract}

\section{General Terms}

AMS Mathematics Classification (2010), 03E72, 68R10, 68R05.

\section{Keywords}

Interval-valued fuzzy graph, domination number

\section{INTRODUCTION}

Fuzzy graph is one of the application tool in the field of mathematics, which allow the users to describe the relationship between any notions easily.

Zadeh [15] was introduced the notion of interval-valued fuzzy sets as an extension of fuzzy sets [14] in which the values of the membership degrees are intervals of numbers instead of the numbers. Interval-valued fuzzy sets provide a more adequate description of uncertainty than traditional fuzzy sets. The fuzzy relations between fuzzy sets were also considered by Rosenfeld [9] and he developed the structure of fuzzy graphs. Recently. Akram and Dudek have studied several properties and operations on interval-valued fuzzy graphs [1]. Some important works in fuzzy graph theory can be found in $\mid 4,5,6,7,8,10,13]$. Somasundaram and Somasundaran [11, 12] introduced and investigated the concept of domination in fuzzy graphs, while P. Debnath [3] introduced and investigated the concept of domination in interval-valued fuzzy graphs. Since the theory of fuzzy sets has become a vigorous area of research in different disciplines including medical and life sciences, management sciences, social sciences engineering, statistic, graph theory, artificial intelligence, signal processing, multi-agent sys- tems, decision making and automate theory and also the domination in fuzzy graphs, this is what motivated us to submit this work. In this paper,we introduced and establish the domination number $\gamma$ of some operation on interval-valued fuzzy graphs, such as union, join, Cartesian product and composition.

\section{PRELIMINARIES}

We review in this section, some basic definitions related to intervalvalued fuzzy graphs and domination in fuzzy graph.

An interval-valued fuzzy graph of a graph $G^{*}=(V, E)$ is a pair $G=(A, B)$, where $A=\left[\mu_{A}^{-}, \mu_{A}^{+}\right]$is an intervalvalued fuzzy set on $V, B=\left[\rho_{B}^{-}, \rho_{B}^{+}\right]$is an interval-valued fuzzy relation on $V$, such that $\mu_{A}^{-}(x) \leq \mu_{A}^{+}(x) ; \forall x \in$ $V$ and $\rho_{B}^{-}(x, y) \leq \min \left\{\mu_{A}^{-}(x), \mu_{A}^{-}(y)\right\}$ and $\rho_{B}^{+}(x, y) \leq$ $\min \left\{\mu_{A}^{+}(x), \mu_{A}^{+}(y)\right\} ; \forall(x, y) \in E$. In an interval-valued fuzzy graph $G$, when $\rho^{-}(u, v)=\rho^{+}(u, v)=0$ for some $u$ and $v$, then there is no edge between $u$ and $v$. Otherwise there exists an edge between $u$ and $v$. Let $G=(A, B)$ be an interval-valued fuzzy graph. Then the cardinality of interval-valued fuzzy graph $G$ is defined as

$|G|=\sum_{u \in V} \frac{1+\mu^{+}(u)-\mu^{-}(v)}{2}+\sum_{(u, v) \in E} \frac{1+\rho^{+}(u, v)-\rho^{-}(u, v)}{2}$.

The vertex cardinality of an interval-valued fuzzy graph $G$ is defined by

$$
|V|=\sum_{u \in V} \frac{1+\mu^{+}(u)-\mu^{-}(u)}{2} .
$$

For all $u \in V$ is called the order of an interval-valued fuzzy graph and is denoted by $p(G)$. The edge cardinality of an interval-valued fuzzy graph $G$ is defined by

$$
|E|=\sum_{(u, v) \in E} \frac{1+\rho^{+}(u, v)-\rho^{-}(u, v)}{2} .
$$


For all $(u, v) \in E$ is called the size of an interval-valued fuzzy graph and is denoted by $q(G)$. An edge $e=(x, y)$ of an interval-valued fuzzy graph is called effective edge if $\rho^{+}(x, y)=$ $\min \left\{\mu^{+}(x), \mu^{+}(y)\right\}$ and $\rho^{-}(x, y)=\min \left\{\mu^{-}(x), \mu^{-}(y)\right\}$. The degree of a vertex can be generalized in different ways for an interval-valued fuzzy graph $G=(A, B)$. The effective degree of a vertex $v$ in an interval-valued fuzzy graph, $G=(A, B)$ is defined to be summation of the weights of the effective edges incident at $v$ and it is denoted by $d_{E}(v)$. The minimum effective edges degree of $G$ is $\delta_{E}(G)=\min \left\{d_{E}(v) \mid v \in V\right\}$. The maximum effective degree of $G$ is $\Delta_{E}(G)=\max \left\{d_{E}(v) \mid v \in V\right\}$. A vertex $u$ of an interval-valued fuzzy graph $G$ is said to be an isolated vertex if $\rho^{-}(u v)<\min \left\{\mu^{-}(u), \mu^{-}(v)\right\}$ and $\rho^{+}(u v)<$ $\min \left\{\mu^{+}(u), \mu^{+}(v)\right\}$ for all $v \in V-\{u\}$ such that there is an edge between $u$ and $v$, i.e., $N(u)=\phi$. A set $S$ of vertices of an intervalvalued fuzzy graph $G$ is said to be independent if $\rho^{-}(u v)<$ $\min \left\{\mu^{-}(u), \mu^{-}(v)\right\}$ and $\rho^{+}(u v)<\min \left\{\mu^{+}(u), \mu^{+}(v)\right\}$ for all $u, v \in S$. An interval-valued fuzzy graph, $G=(A, B)$ is said to be Complete interval-valued fuzzy graph if $\rho^{-}\left(v_{i}, v_{j}\right)=$ $\min \left\{\mu^{-}\left(v_{i}\right), \mu^{-}\left(v_{j}\right)\right\}, \rho^{+}(u, v)=\min \left\{\mu^{+}(u), \mu^{+}(v)\right\}$, for all $u, v \in V$ and denoted by $K_{p}$. The complement of an intervalvalued fuzzy graph, $G=(A, B)$ is an interval-valued fuzzy graph, $\bar{G}=(\bar{A}, \bar{B})$ where

$\overline{\rho^{-}}(u, v)=\min \left\{\mu^{-}(u), \mu^{-}(v)\right\}-\rho^{-}(u, v)$ and $\overline{\rho^{+}}(u, v)=$ $\min \left\{\mu^{+}(u), \mu^{+}(v)\right\}-\rho^{+}(u, v)$ for all $u, v \in G$. An intervalvalued fuzzy graph $G=(A, B)$ of a graph $G^{*}=(V, E)$ is said to be bipartite if the vertex set $V$ can be partitioned into two nonempty sets $V_{1}$ and $V_{2}$ such that $\rho^{-}(x y)=0$ and $\rho^{+}(x y)=0$ if $x, y \in V_{1}$ or $x, y \in V_{2}$. Further if $\rho^{-}(x y)=\min \left\{\mu_{A}^{-}(x), \mu_{A}^{-}(y)\right\}$ and $\rho^{+}(x y)=\min \left\{\mu_{A}^{+}(x), \mu_{A}^{+}(y)\right\}$ for all $x \in V_{1}$ and $y \in V_{2}$. Then $G$ is called a complete bipartite fuzzy graph is denoted by $K_{\mu_{A}^{-}, \mu_{A}^{+}}$, where $\mu_{A}^{-}$and $\mu_{A}^{+}$are restrictions of $\mu_{A}^{-}$and $\mu_{A}^{+}$on $V_{1}$ and $V_{2}$ respectively. An edge $e=x y$ of an interval-valued fuzzy graph $G$ is called an effective edge if $\rho^{-}(x y)=\min \left\{\mu_{A}^{-}(x), \mu_{A}^{-}(y)\right\}$ and $\rho^{+}(x y)=\min \left\{\mu_{A}^{+}(x), \mu_{A}^{+}(y)\right\}$. In this case, the vertex $x$ is called a neighbor of $\mathrm{y}$ and conversely. $N(x)=\{y \in V$ : $y$ isaneighbourof $x\}$ is called the neighborhood of $x$. A subset ( $S \subseteq V$ ) of $V(G)$ is called dominating set in interval-valued fuzzy graph $G$, if for every $v \in V-S$ there exists $u \in S$ Such that $(u, v)$ is strong edge. A dominating set $S$ of $G$ is called minimal dominating set of $G$ if $S-\{u\}$ is not dominating set for every $u \in S$. A minimal dominating set $S$, with $|S|=\gamma(G)$ is denoted by $\gamma-$ set.

\section{MAIN RESULTS}

Definition 1. [1] Let $G_{1}=\left(A_{1}, B_{1}\right)$ and $G_{2}=\left(A_{2}, B_{2}\right)$ be two interval-valued fuzzy graph on $A_{1}, A_{2}$, respectively with $A_{1} \cap A_{2}=\phi$. The union of $G_{1}$ and $G_{2}$ is the interval-valued fuzzy graph $G_{1} \cup G_{2}$ on $A_{1} \cup A_{2}$ and defined as $G=G_{1} \cup G_{2}=$ $\left\{\left(\mu_{A_{1}^{-}} \cup \mu_{A_{2}^{-}}\right),\left(\mu_{A_{1}^{+}} \cup \mu_{A_{2}^{+}}\right),\left(\rho_{B_{1}^{-}} \cup \rho_{B_{2}^{-}}\right),\left(\rho_{B_{1}^{+}} \cup \rho_{B_{2}^{+}}\right)\right\}$. Where

$$
\begin{gathered}
\left(\mu_{A_{1}^{-}} \cup \mu_{A_{2}^{-}}\right)(u)=\left\{\begin{array}{lll}
\mu_{A_{1}^{-}} & \text {if } & u \in A_{1} \\
\mu_{A_{2}^{-}} & \text {if } & u \in A_{2}
\end{array}\right. \\
\left(\mu_{A_{1}^{+}} \cup \mu_{A_{2}^{+}}\right)(u)=\left\{\begin{array}{lll}
\mu_{A_{1}^{+}} & \text {if } & u \in A_{1} \\
\mu_{A_{2}^{+}} & \text {if } & u \in A_{2}
\end{array}\right. \\
\left(\rho_{B_{1}^{-}} \cup \rho_{B_{2}^{-}}\right)(u v)=\left\{\begin{array}{lll}
\rho_{B_{1}^{-}} & \text {if } & u v \in B_{1} \\
\rho_{B_{2}^{-}} & \text {if } & u v \in B_{2}
\end{array}\right.
\end{gathered}
$$

$$
\left(\rho_{B_{1}^{+}} \cup \rho_{B_{2}^{+}}\right)(v u)=\left\{\begin{array}{lll}
\rho_{B_{1}^{+}} & \text {if } & u v \in B_{1} \\
\rho_{B_{2}^{+}} & \text {if } & u v \in B_{2}
\end{array}\right.
$$

In the flowing we given $\gamma$ of the union of every tow interval-valued fuzzy graphs $G_{1}$ and $G_{2}$.

THEOREM 2. Let $G_{1}$ and $G_{2}$ be two vertices disjoint intervalvalued fuzzy graphs, then

$$
\gamma\left(G_{1} \cup G_{2}\right)=\gamma\left(G_{1}\right)+\gamma\left(G_{2}\right) .
$$

Proof. Let $S_{1}$ and $S_{2}$ be tow $\gamma_{1}-$ set and $\gamma_{2}$ of $G_{1}$ and $G_{2}$ respectively. Since $G_{1}$ and $G_{2}$ are vertices disjoint.Then $V_{1} \cap V_{2}=$ $\phi$ and $V_{1} \cup V_{2}=V$. Therefore, $S_{1} \cup S_{2}$, is dominating set of $\left(G_{1} \cup G_{2}\right)$. Hence,

$$
\gamma\left(G_{1} \cup G_{2}\right)=\left|S_{1} \cup S_{2}\right|=\gamma\left(G_{1}\right)+\gamma\left(G_{2}\right) .
$$

The following example discusses in detail the result in the above Theorem.

EXAMPLE 1. Consider the interval-valued fuzzy graphs $G_{2}$ and $G_{1}$ given in the Figures $3.1 a$ and $3.1 b$, respectively, such that all edges of $G_{1}$ and $G_{2}$ are strong edges.

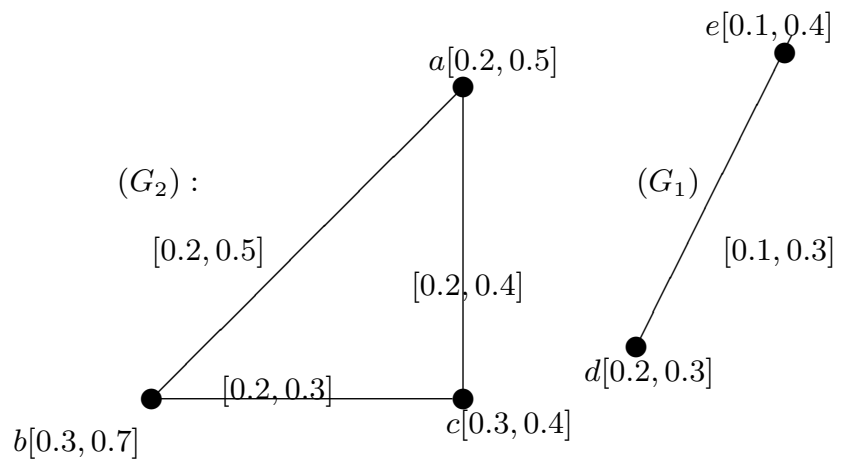

Fig $3.1 a$

Fig 3.1b.

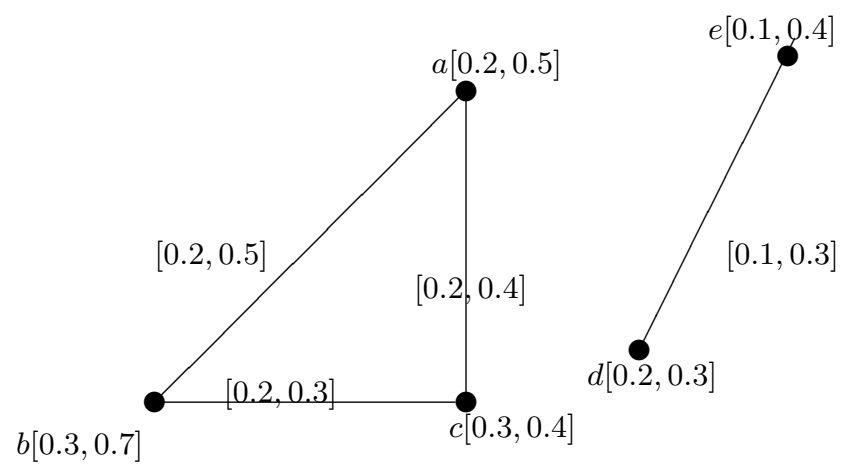

Fig $3.1 c:\left(G_{1} \cup G_{2}\right)$ 
The union of $G_{1}$ and $G_{2}$ is shown in Figure $(3.1 c$,$) we see that$ $S_{1}=\{a\}$ is a $\gamma-$ set of $G_{1}, S_{2}=\{d\}$ is a $\gamma-$ set of $G_{2}$ and $\gamma_{1}=0.65, \gamma_{2}=0.55$. Now, $S=S_{1} \cup S_{2}$ is a $\gamma-$ set of $G_{1} \cup G_{2}$. Hence $\gamma\left(G_{1} \cup G_{2}\right)=\left|S_{1} \cup S_{2}\right|=\left|S_{1}+S_{2}\right|=0.65+0.55=$ $1.2=\gamma_{1}+\gamma_{2}$.

Definition 3. [1] The join $G_{1}+G_{2}=\left(A_{1}+A_{2}, B_{1}+\right.$ $B_{2}$ ) of two produce interval-valued fuzzy graphs $G_{1}$ and $G_{2}$ is defined as follows:

$\left(\mu_{A_{1}^{-}}+\mu_{A_{2}^{-}}\right)(u)=\left(\mu_{A_{1}^{-}} \cup \mu_{A_{2}^{-}}\right)(u)$,

$\left(\mu_{A_{1}^{+}}+\mu_{A_{2}^{+}}\right)(u)=\left(\mu_{A_{1}^{+}} \cup \mu_{A_{2}^{+}}\right)(u)$ if $u \in V_{1} \cup V_{2}$

$\left(\rho_{B_{1}^{-}}+\rho_{B_{2}^{-}}\right)(u v)=\left(\rho_{B_{1}^{-}} \cup \rho_{B_{2}^{-}}\right)(u v)$,

$\left(\rho_{B_{1}^{+}}+\rho_{B_{2}^{+}}\right)(u v)=\left(\rho_{B_{1}^{+}} \cup \rho_{B_{2}^{+}}(u v)\right.$ if $u v \in E_{1} \cap E_{2}$.

$\left(\rho_{B_{1}^{-}}+\rho_{B_{2}^{-}}\right)(u v)=\min \left(\mu_{A_{1}^{-}}(u), \mu_{A_{2}^{-}}(v)\right)$,

$\left(\rho_{B_{1}^{+}}+\rho_{B_{2}^{+}}\right)(u v)=\left(\mu_{A_{1}^{+}}(u), \mu_{A_{2}^{+}}(v)\right)$ if $u v \in E$

where $E$ is the set of all edges joining the vertices of $V_{1}$ and $V_{2}$.

The flowing Theorem given $\gamma$ of $G_{1}+G_{2}$.

THEOREM 4. Let $G_{1}$ and $G_{2}$ be any two interval-valued fuzzy graphs, then

$$
\gamma\left(G_{1}+G_{2}\right)=\min \left\{\gamma\left(G_{1}\right), \gamma\left(G_{2}\right)\right\}
$$

Proof. First we prove both $S_{1}$ and $S_{2}$ are dominating sets of $G_{1}+G_{2}$. By definition of join of tow interval-valued fuzzy graphs $G_{1}$ and $G_{2}$. Then for-all each vertex $v \in V\left(G_{1}\right)$ and $u \in V\left(G_{2}\right)$, we have $u v \in G_{1} \cup G_{2}$ is a strong edge. Since $S_{1} \subseteq V_{1}$. Then $\forall u \in V\left(G_{1} \cup G_{2}\right) \exists u \in S_{1}$, such that $u v$ is a strong edge. Therefore, $S_{1}$ is dominating set of $G_{1} \cup G_{2}$. Similarly $S_{2}$ is dominating set of $G_{1} \cup G_{2}$. Hence $\gamma\left(G_{1} \cup G_{2}\right)=\min \left\{S_{1}, S_{2}\right\}=$ $\min \left\{\gamma\left(G_{1}\right), \gamma\left(G_{2}\right)\right\}$. If $y \in V\left(G_{1}\right)$ obviously is dominating by a $x \in S_{1}$, since $S_{1}$ is the dominating set of $G_{1}$, if $y \in G_{2}$, now we prove $x \in S_{1}$ dominates $y$. Since $x \in G_{1}$ and $y \in G_{2}$, then $\left(\rho_{B_{1}^{-}}+\rho_{B_{2}^{-}}\right)(x y)=\min \left(\mu_{A_{1}^{-}}(x), \mu_{A_{2}^{-}}(y)\right)$,

$\left(\rho_{B_{1}^{+}}+\rho_{B_{2}^{+}}\right)(x y)=\left(\mu_{A_{1}^{+}}(x), \mu_{A_{2}^{+}}(y)\right)$, clearly $x$ dominates $y$. Therefore, $S_{1}$ is the dominating set of $G_{1}+G_{2}$. We assume $S_{1}$ is not minimum dominating set of $G_{1}+G_{2}$, there exist $x \in S_{1}$ such that $S_{1}-x$ is a dominating set of $G_{1}+G_{2}$. Therefore $x \in G_{1}+G_{2}$, there exist $y \in S_{1}$ such that $\left(\rho_{B_{1}^{-}}+\rho_{B_{2}^{-}}\right)(x y)=$ $\min \left(\mu_{A_{1}^{-}}(x), \mu_{A_{2}^{-}}(y)\right)$,

$\left(\rho_{B_{1}^{+}}+\rho_{B_{2}^{+}}\right)(x y)=\left(\mu_{A_{1}^{+}}(x), \mu_{A_{2}^{+}}(y)\right)$, this implies $S_{1}-x$ is the dominating set of $G_{1}$, this is contradicting to is $S_{1}$ is minimum.

Similarly: we prove $S_{2}$ is a minimum dominating set of $G_{1}+G_{2}$. Let $S$ be the minimum dominating set of $G_{1}+G_{2}$. Then $|S|=$ $\left|S_{1}+S_{2}.\right|$ Hence

$$
\gamma\left(G_{1}+G_{2}\right)=\min \left\{\gamma\left(G_{1}\right), \gamma\left(G_{2}\right)\right\}
$$

The following example discusses in detail the result in the above Theorem.

EXAMPLE 2. Consider the interval-valued fuzzy graphs $G_{1}$ and $G_{2}$ given in the Figures $3.2 a$ and $3.2 b$ respectively, such that

all edges in $G_{1}$ and $G_{2}$ are effective.

$v_{1}[0.2,0.3]$

$u_{1}[0.1,0.3]$

$u_{2}[0.2,0.3]$

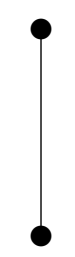

$G_{1}$ :

$u_{1}[0.1,0.3]$

$u_{2}[0.2,0.3]$

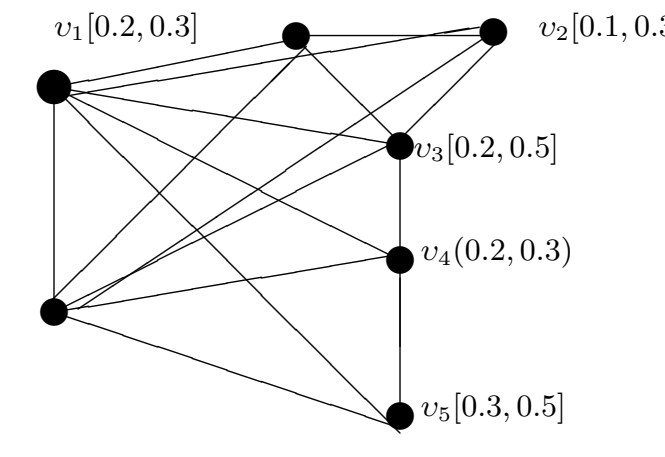

$G_{1}+G_{2}$

Fig $3.2 b$

The join of $G_{1}$ and $G_{2}$ given in Figure $(3.2 b)$ we can verify that $S_{1}=\left\{u_{2}\right\}$ is a $\gamma-$ set of $G_{1}, S_{2}=\left\{v_{1}, v_{2}\right\}$ is a $\gamma-$ set of $G_{2}$ and $S=S_{1} \quad$ or $S=S_{2}$ is a dominating set of $G_{1}+G_{2}$. Thus $\gamma\left(G_{1}+G_{2}\right)=\min \left\{S_{2}, S_{2}\right\}=\{f\}=0.55$

Definition 5. [1] Let $G_{1}=\left(A_{1}, B_{1}\right)$ and $G_{2}=\left(A_{2}, B_{2}\right)$ be two interval-valued fuzzy graphs on $A_{1}, A_{2}$, respectively with $A_{1} \cap A_{2}=\phi$. The Cartesian product of $G_{1}$ and $G_{2}$ defined by $G_{1} \times G_{2}$ is the interval-valued fuzzy graph $G=G_{1} \times G_{2}$ on $A_{1} \times A_{2}$ and defined by $G=G_{1} \times G_{2}=$ $\left\{\left(\mu_{A_{1}^{-}} \times \mu_{A_{2}^{-}}, \mu_{A_{1}^{+}} \times \mu_{A_{2}^{+}}\right),\left(\rho_{B_{1}^{-}} \times \rho_{B_{2}^{-}}, \rho_{B_{1}^{+}} \times \rho_{B_{2}^{+}}\right)\right\}$, such

$$
\begin{aligned}
& \text { that }\left(\mu_{A_{1}^{-}} \times \mu_{A_{2}^{-}}\right)(x y)=\min \left\{\mu_{A_{1}^{-}}(x), \mu_{A_{2}^{-}}(y)\right\} \\
& \left\{\begin{array}{l}
\left(\mu_{A_{1}} \times \mu_{A_{2}^{+}}\right)(x y)=\min \left\{\mu_{A_{1}^{+}}(x), \mu_{A_{2}^{+}}(y)\right\}
\end{array} \quad \forall x, y \in A_{1} \times A_{2}\right. \\
& \left\{\begin{array}{rl}
\left(\rho_{B_{1}^{-}} \times \rho_{B_{2}^{-}}\right)\left(\left(x y_{1}\right)\left(x y_{2}\right)\right) & =\min \left\{\mu_{A_{1}^{-}}(x), \rho_{B_{2}^{-}}\left(y_{1} y_{2}\right)\right\} \\
\left(\rho_{B_{1}^{+}} \times \rho_{B_{2}^{+}}\right)\left(\left(x y_{1}\right)\left(x y_{2}\right)\right) & =\min \left\{\mu_{A_{1}^{+}}(x), \rho_{B_{2}^{+}}\left(y_{1} y_{2}\right)\right\}
\end{array} \quad \forall x \in A_{1}, y_{1}\right. \\
& \left\{\begin{array}{rl}
\left(\rho_{B_{1}^{-}} \times \rho_{B_{2}^{-}}\right)\left(\left(x_{1} y\right)\left(x_{2} y\right)\right) & =\min \left\{\rho_{B_{1}^{-}}\left(x_{1} x_{2}\right), \mu_{A_{2}^{-}}(y)\right\} \\
\left(\rho_{B_{1}^{+}} \times \rho_{B_{2}^{+}}\right)\left(\left(x_{1} y\right)\left(x_{2} y\right)\right) & =\min \left\{\rho_{B_{1}^{+}}\left(x_{1} x_{2}\right), \mu_{A_{2}^{+}}(y)\right\}
\end{array} \quad \forall y \in A_{2}, x_{1}\right.
\end{aligned}
$$


The flowing Theorem given $\gamma$ for the cartisian product of any tow interval-valued fuzzy graphs $G_{1}$ and $G_{2}$.

THEOREM 6. Let $S_{1}$ and $S_{2}$ be $\gamma-$ set of interval-valued fuzzy graphs $G_{1}$ and $G_{2}$, respectively. Then

$$
\gamma\left(G_{1} \times G_{2}\right)=\min \left\{\left|S_{1} \times V_{2}\right|,\left|V_{1} \times S_{2}\right|\right\} .
$$

Proof. First we prove that $S_{1} \times V_{2}$ is a dominating set of $\left(G_{1} \times G_{2}\right)$. Let $\left(u_{1}, u_{2}\right) \notin S_{1} \times V_{2}$. Hence $u_{1} \notin S_{1}$. Since $S_{1}$ is dominating set of $G_{1}$. Then there exist a vertex $v_{1} \in S_{1}$, such that $\forall u_{1} \in V_{1}-S_{1}\left\{\begin{array}{l}\rho_{B}^{-}\left(u_{1}, v_{1}\right)=\min \left\{\mu_{A}^{-}\left(u_{1}\right), \mu_{A}^{-}\left(v_{1}\right)\right\} \\ \rho_{B}^{+}\left(u_{1}, v_{1}\right)=\min \left\{\mu_{A}^{+}\left(u_{1}\right), \mu_{A}^{+}\left(v_{1}\right)\right\}\end{array}\right.$ $\left(v_{1}, u_{2}\right) \in S_{1} \times V_{2}$,

$$
\rho_{B}^{-}\left(\left(u_{1}, u_{2}\right),\left(v_{1}, u_{2}\right)\right)=\min \left\{\rho_{B_{1}}^{-}\left(u_{1} v_{1}\right), \mu_{A_{2}}^{-}\left(u_{2}\right)\right\}
$$

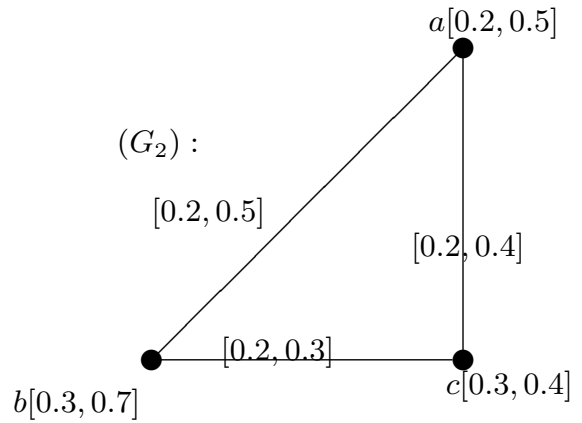

Fig $3.3 a$

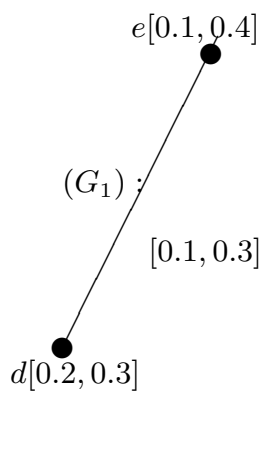

Fig $3.3 b$

$$
\begin{aligned}
& =\min \left\{\mu_{A_{1}}^{-}\left(u_{1}\right) \wedge \mu_{A_{1}}^{-}\left(v_{1}\right), \mu_{A_{2}}^{-}\left(u_{2}\right)\right\}=\min \left\{\mu_{A_{1}}^{-}\left(u_{1}\right) \wedge \mu_{A_{1}}^{-}\left(v_{1}\right) \wedge \mu_{A_{2}}^{-}\left(u_{2}\right)\right\} \\
& =\min \left\{\mu_{A_{1}}^{-}\left(u_{1}\right) \wedge \mu_{A_{2}}^{-}\left(u_{2}\right)\right\}, \min \left\{\mu_{A_{2}}^{-}\left(u_{2}\right) \wedge \mu_{A_{1}}^{-}\left(v_{1}\right)\right\}=\rho_{B}^{-}\left(( u _ { 1 } , u _ { 2 } ) \wedge \rho _ { B } ^ { - } \left(\left(v_{1}, u_{2}\right)\right.\right. \\
& \quad \rho_{B}^{+}\left(\left(u_{1}, u_{2}\right),\left(\left(v_{1}, u_{2}\right)\right)=\min \left\{\rho_{B_{1}}^{+}\left(u_{1} v_{1}\right), \mu_{A_{2}}^{+}\left(u_{2}\right)\right\}\right. \\
& =\min \left\{\mu_{A_{1}}^{+}\left(u_{1}\right) \wedge \mu_{A_{1}}^{+}\left(v_{1}\right), \mu_{A_{2}}^{+}\left(u_{2}\right)\right\}=\min \left\{\mu_{A_{1}}^{+}\left(u_{1}\right) \wedge \mu_{A_{1}}^{+}\left(v_{1}\right) \wedge \mu_{A_{2}}^{+}\left(u_{2}\right)\right\} \\
& =\min \left\{\mu_{A_{1}}^{+}\left(u_{1}\right) \wedge \mu_{A_{2}}^{+}\left(u_{2}\right)\right\}, \min \left\{\mu_{A_{2}}^{+}\left(u_{2}\right) \wedge \mu_{A_{1}}^{+}\left(v_{1}\right)\right\}=\rho_{B}^{+}\left(( u _ { 1 } , u _ { 2 } ) \wedge \rho _ { B } ^ { + } \left(\left(v_{1}, u_{2}\right)\right.\right.
\end{aligned}
$$

This implies every $\left(u_{1}, u_{2}\right) \notin S_{1} \times V_{2}$, there exist a vertex $\left(v_{1}, u_{2}\right) \in S_{1} \times V_{2}$, such that $\left(u_{1}, u_{2}\right)\left(v_{1}, u_{2}\right)$ is a strong edge. Therefore $S_{1} \times V_{2}$ is dominating set of $G_{1} \times G_{2}$. Similarly; $V_{1} \times S_{2}$ is dominating set of $G_{1} \times G_{2}$. Next we prove that $V_{1} \times S_{2}$ and $S_{1} \times V_{2}$ are a minimal dominating sets. Suppose $S_{1} \times V_{2}$ is not minimal dominating set of $G_{1} \times G_{2}$. Then there exist a vertex $\left(x_{1}, u_{2}\right) \in S_{1} \times V_{2}$, such that $\left\{\left\{S_{1} \times V_{2}\right\}-\left(x_{1}, u_{2}\right)\right\}$ is a dominating set of $G_{1} \times G_{2}$. Let $\left(u_{1}, u_{2}\right) \in S_{1} \times V_{2}$, and $\left(u_{1}, u_{2}\right)$ dominates $\left(x_{1}, u_{2}\right)$ and since $S_{1} \times V_{2}$ is dominating set of $G_{1} \times G_{2}$. Then we get $u_{1}$ dominates $x_{1} \in S_{1}$. Clearly $S_{1}-\left\{x_{1}\right\}$ is a dominating set of $G_{1}$, which a contradiction to our assumption $S_{1}$ is a minimal dominating set of $G_{1}$. Therefore $S_{1} \times V_{2}$ is minimal dominating set of $G_{1} \times G_{2}$. Similarly; $V_{1} \times S_{2}$ is minimal dominating set of $G_{1} \times G_{2}$. Hence

$$
\gamma\left(G_{1} \times G_{2}\right)=\min \left\{\left|S_{1} \times V_{2}\right|,\left|V_{1} \times S_{2}\right|\right\} .
$$

The following example discusses in detail the result in the above Theorem.

EXAMPLE 3. Consider the interval-valued fuzzy graphs $G_{2}$ and $G_{1}$ given in the Figures $3.3 a$ and $3.3 b$.
$[0.2,0.3]$

$c d[0.2,0$

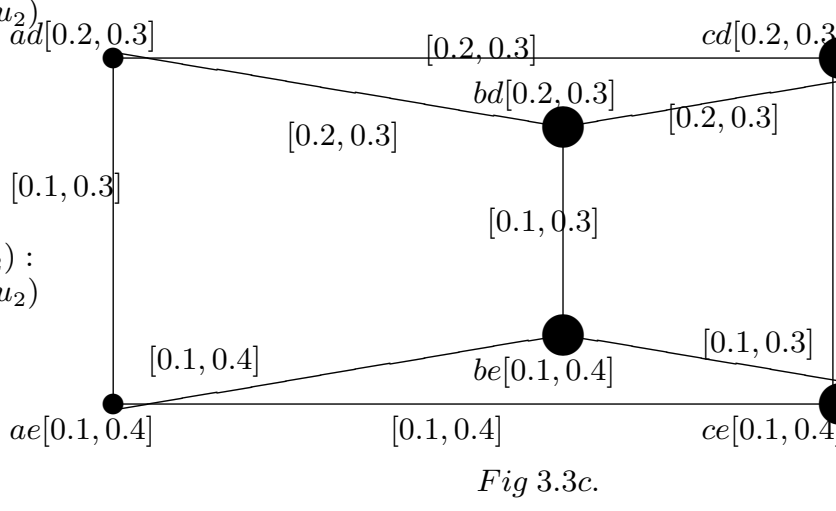

From Figures (3.3a) and (3.3b), we can verify that $S_{1}=\{d\}$ is a $\gamma-$ set of $G_{1}$ and $S_{2}=\{a\}$ is a $\gamma-$ set of $G_{2}$. Also we can verify that from Figure $(3.3 \mathrm{c}),\left(V_{1} \times S_{2}\right)$ and $\left(V_{2} \times S_{1}\right)$ are dominating sets of $G_{1} \times G_{2}$. Hence

$\gamma\left(G_{1} \times G_{2}\right)=\min \left\{\left|S_{1} \times V_{2}\right|,\left|V_{1} \times S_{2}\right|\right\}=\min \{|\{a d, a e\}|,|\{a d, b d, c d\}|\}$

Definition 7. [1] Let $G_{1}=\left(A_{1}, B_{1}\right)$ and $G_{2}=\left(A_{2}, B_{2}\right)$ be two interval-valued fuzzy graphs on $A_{1}, A_{2}$, respectively with $A_{1} \cap A_{2}=\phi$. The composition of $G_{1}$ and $G_{2}$ defined by $G_{1} \circ G_{2}$ is the interval-valued fuzzy graph $G=G_{1} \circ G_{2}$ on $A_{1} \circ A_{2}$ and defined by $G=G_{1} \circ G_{2}=$ $\left\{\left(\mu_{A_{1}^{-}} \circ \mu_{A_{2}^{-}}, \mu_{A_{1}^{+}} \circ \mu_{A_{2}^{+}}\right),\left(\rho_{B_{1}^{-}} \circ \rho_{B_{2}^{-}}, \rho_{B_{1}^{+}} \circ \rho_{B_{2}^{+}}\right)\right\}$, such that $\left\{\begin{array}{c}\left(\mu_{A_{1}^{-}} \circ \mu_{A_{2}^{-}}\right)(x y)=\min \left\{\mu_{A_{1}^{-}}(x), \mu_{A_{2}^{-}}(y)\right\} \\ \left(\left(\mu_{A_{1}^{+}} \circ \mu_{A_{2}^{+}}\right)(x y)=\min \left\{\mu_{A_{1}^{+}}(x), \mu_{A_{2}^{+}}(y)\right\}\right.\end{array} \forall x, y \in A_{1} \times A_{2}\right.$ $\left\{\begin{array}{rl}\left(\rho_{B_{1}^{-}} \circ \rho_{B_{2}^{-}}\right)\left(\left(x y_{1}\right)\left(x y_{2}\right)\right) & =\min \left\{\mu_{A_{1}^{-}}(x), \rho_{B_{2}^{-}}\left(y_{1} y_{2}\right)\right\} \\ \left(\rho_{B_{1}^{+}} \circ \rho_{B_{2}^{+}}\right)\left(\left(x y_{1}\right)\left(x y_{2}\right)\right) & =\min \left\{\mu_{A_{1}^{+}}(x), \rho_{B_{2}^{+}}\left(y_{1} y_{2}\right)\right\}\end{array} \quad \forall x \in A_{1}, y_{1} y\right.$ $\left\{\begin{array}{rl}\left(\rho_{B_{1}^{-}} \circ \rho_{B_{2}^{-}}\right)\left(\left(x_{1} y\right)\left(x_{2} y\right)\right) & =\min \left\{\rho_{B_{1}^{-}}\left(x_{1} x_{2}\right), \mu_{A_{2}^{-}}(y)\right\} \\ \left(\rho_{B_{1}^{+}} \circ \rho_{B_{2}^{+}}\right)\left(\left(x_{1} y\right)\left(x_{2} y\right)\right) & =\min \left\{\rho_{B_{1}^{+}}\left(x_{1} x_{2}\right), \mu_{A_{2}^{+}}(y)\right\}\end{array} \quad \forall y \in A_{2}, x_{1}\right.$ 
$\left\{\begin{array}{rl}\left(\rho_{B_{1}^{-}} \circ \rho_{B_{1}^{-}}\right)\left(\left(x_{1} y_{1}\right)\left(x_{2} y_{2}\right)\right) & =\min \left\{\rho_{B_{1}^{-}}\left(x_{1} x_{2}\right), \mu_{A_{2}^{-}}\left(y_{1} \wedge \mu_{A_{2}^{-}}\left(y_{2} \text { and }\right)\right.\right. \\ \left(\rho_{B_{1}^{+}} \circ \rho_{B_{2}^{+}}\right)\left(\left(x_{1} y_{1}\right)\left(x_{2} y_{2}\right)\right) & =\min \left\{\rho_{B_{1}^{+}}\left(x_{1} x_{2}\right), \mu_{A_{2}^{+}}\left(y_{1}\right) \wedge \mu_{A_{2}^{+}}\left(y_{2}\right)\right\}\end{array} \quad \forall\left(y_{1} y_{2}\right) \in A_{2},\left(x_{1} x_{2}\right) \in B_{1}\right.$.

Such that $\left(y_{1} \neq y_{2}\right)$.

$\rho_{B^{-}}\left(v, v_{1}\right)=\min \left(\mu_{A_{1}^{-}}(v), \mu_{A_{2}^{-}}\left(v_{1}\right)\right), \rho_{B^{+}}\left(v, v_{1}\right)=\min \left(\mu_{A_{1}^{+}}(v), \mu_{A_{2}^{+}}(v\right.$

THEOREM 8. Let $S_{1}$ and $S_{2}$ be $\gamma-$ set of interval-valued fuzzy graphs $G_{1}$ and $G_{2}$, respectively. Then

$$
\gamma\left(G_{1} \circ G_{2}\right)=\left|S_{1} \times S_{2}\right| .
$$

Let $\left(u_{2}, v_{2}\right) \in S_{1} \times S_{2}$. Then

Proof. Let $(u, v) \in S_{1} \times S_{2}$. Then we have three cases:

Case 1: $u \notin S_{1}$ and $v \in S_{2}$. Let $u \notin S_{1}$. Then there exist $u_{1} \in S_{1}$, such that $u_{1}$ dominates $u$. Hence

$$
\begin{aligned}
& \left\{\begin{array}{l}
\rho_{B_{1}}^{-}\left(u_{1}, u\right)=\min \left\{\mu_{A_{1}}^{-}\left(u_{1}\right), \mu_{A_{2}}^{-}(u)\right\} \\
\rho_{B_{1}}^{+}\left(u_{1}, u\right)=\min \left\{\mu_{A_{1}}^{+}\left(u_{1}\right), \mu_{A_{2}}^{+}(u)\right\}
\end{array} \quad \text { Now }\left(u_{1}, v\right) \in S_{1} \times \quad=\left(\mu_{A_{1}} \circ \mu_{A_{2}}\right)^{-}(u, v) \wedge\left(\mu_{A_{1}} \circ \mu_{A_{2}}\right)^{-}\left(u_{1}, v_{2}\right)=\min \left(\mu_{A}^{-}(u, v), \mu_{A}^{-}\left(u_{1}, v_{2}\right) .\right.\right. \\
& S_{2} \\
& \rho_{B_{1}}^{-}\left(\left(u_{1}, u\right),\left(v, u_{1}\right)\right)=\rho_{B_{1}}^{-}\left(u_{1}, u\right) \wedge \mu_{A_{2}}^{-}(v)=\min \left\{\rho_{B_{1}}^{-}\left(u_{1}, u\right), \mu_{A_{2}}^{-}(v)\right\} \quad \rho_{B^{+}}\left((u, v),\left(u_{1}, v_{2}\right)\right)=\min \left(\mu_{A_{2}^{+}}(v), \mu_{A_{2}^{+}}\left(v_{1}\right),\right. \\
& =\min \left\{\mu_{A_{1}}^{-}\left(u_{1}\right) \wedge \mu_{A_{1}}^{-}\left(v_{1}\right), \mu_{A_{2}}^{-}\left(u_{2}\right)\right\}=\mu_{A_{1}}^{-}\left(u_{1}\right) \wedge \mu_{A_{2}}^{-}(v) \wedge \mu_{A_{2}}^{-}(v) \wedge \mu_{A_{1}^{-}}\left(u_{B_{1}}\right) \\
& =\left(\mu_{A_{1}^{+}}^{-} \circ \mu_{A_{2}^{-}}\right)^{-}(u, v) \wedge\left(\mu_{A_{1}}^{-} \mu_{A_{2}^{-}}\right)^{-}\left(u_{1}, v\right)=\left(\mu_{A_{2}^{+}}(v), \mu_{A_{2}^{+}}\left(v_{1}\right), \mu_{A_{1}^{+}}(u) \wedge \mu_{A_{1}^{+}}\left(u_{1}\right)\right) \\
& =\left(\mu_{A_{1}} \circ \mu_{A_{2}}\right)^{-}(u, v) \wedge\left(\mu_{A_{1}} \circ \mu_{A_{2}}\right)^{-}\left(u_{1}, v\right) \\
& \text { and }=\left(\mu_{A_{1}} \circ \mu_{A_{2}}\right)^{+}(u, v) \wedge\left(\mu_{A_{1}} \circ \mu_{A_{2}}\right)^{+}\left(u_{1}, v_{2}\right)
\end{aligned}
$$$$
\rho_{B^{-}}\left((u, v),\left(u_{1}, v_{2}\right)\right)=\min \left(\rho_{A_{2}^{-}}(v), \mu_{A_{2}^{-}}\left(v_{1}\right), \rho_{B_{1}^{-}}\left(u, u_{1}\right)\right)=\left(\mu_{A_{2}^{-}}(v), \mu\right.
$$

$\rho_{B}^{+}\left(\left(u_{1}, u\right),\left(v, u_{1}\right)\right)=\rho_{B_{1}}^{+}\left(u_{1}, u\right) \wedge \mu^{+}(v)=\min \left\{\rho_{B_{1}}^{+}\left(u_{1}, u\right), \mu^{+}(v)^{\text {Therefore, }}\left(u_{1}, v_{2}\right)\right.$ dominates $(u, v)$ in $G_{1} \circ G_{2}$, this implies $S_{1} \times$ $\rho_{B}^{+}\left(\left(u_{1}, u\right),\left(v, u_{1}\right)\right)=\rho_{B_{1}}^{+}\left(u_{1}, u\right) \wedge \mu_{A_{2}}^{+}(v)=\min \left\{\rho_{B_{1}}^{+}\left(u_{1}, u\right), \mu_{A_{2}}^{+}(v)_{S_{2}}\right.$ is a dominating set of $G_{1} \circ G_{2}$.

$=\min \left\{\mu_{A_{1}}^{+}\left(u_{1}\right) \wedge \mu_{A_{1}}^{+}\left(v_{1}\right), \mu_{A_{2}}^{+}\left(u_{2}\right)\right\}=\mu_{A_{1}}^{+}\left(u_{1}\right) \wedge \mu_{A_{2}}^{+}(v) \wedge \mu_{A_{2}}^{+}(v) \wedge$ Now, we prove $S_{1} \times S_{2}$ is minimal. Let $(x, y) \in S_{1} \times S_{2}, x \in S_{1}$

$$
\begin{aligned}
& =\left(\mu_{A_{1}}^{+} \circ \mu_{A_{2}^{+}}\right)^{+}(u, v) \wedge\left(\mu_{A_{1}}^{+} \circ \mu_{A_{2}^{+}}\right)^{+}\left(u_{1}, v\right) \\
& =\left(\mu_{A_{1}} \circ \mu_{A_{2}}\right)^{+}(u, v) \wedge\left(\mu_{A_{1}} \circ \mu_{A_{2}}\right)^{+}\left(u_{1}, v\right) .
\end{aligned}
$$

Hence $\left(u_{1}, v\right)$ dominates $(u, v)$.

Case 2: $u \in S_{1}$ and $v \notin S_{2}$. Let $v_{2} \in S_{2}$, such that $\quad\left\{\begin{array}{l}\rho_{B_{2}}^{-}\left(v_{2}, v\right)=\min \left\{\mu_{A_{1}}^{-}\left(v_{2}\right), \mu_{A_{2}}^{-}(v)\right\} \\ \rho_{B_{2}}^{+}\left(v_{2}, v\right)=\min \left\{\mu_{A_{1}}^{+}\left(v_{2}\right), \mu_{A_{2}}^{+}(v)\right\}\end{array} \quad\right.$ Now $\left(u, v_{2}\right) \in S_{1} \times S_{2}$ $\mu_{\text {and }}\left(z_{1} 1 \notin S_{2}\right.$ and since $S_{1}$ and $S_{2}$ are minimal dominating sets of $G_{1}$ and $G_{2}$, respectively.

Therefore, $S_{1}-\{x\}$ and $S_{2}-\{y\}$ are not a dominating sets of $G_{1}$ and $G_{2}$, respectively and then $\left(S_{1} \times S_{2}\right)-(x \times y)$ is not dominating set of $G_{1} \circ G_{2}$, this implies that $S_{1} \times S_{2}$ minimal dominating set of $G_{1} \circ G_{2}$.

The flowing Example explain the results in above Theorem.

EXAMPLE 4. For the interval-valued fuzzy graphs $G_{2}$ and $G_{1}$ given in the Figures $3.4 a$ and $3.4 b$, respectively.

$$
\begin{gathered}
=\min \left\{\mu_{A_{1}}^{-}(u) \wedge \mu_{A_{2}}^{-}(v), \mu_{A_{2}}^{-}\left(v_{2}\right)\right\}=\mu_{A_{1}}^{-}(u) \wedge \mu_{A_{2}}^{-}(v) \wedge \mu_{A_{1}}^{-}(u) \wedge \mu_{A_{2}^{-}}\left(v_{2}\right) \\
=\left(\mu_{A_{1}}^{-} \circ \mu_{A_{2}^{-}}\right)^{-}(u, v) \wedge\left(\mu_{A_{1}}^{-} \circ \mu_{A_{2}^{-}}\right)^{-}\left(u, v_{2}\right) \\
=\left(\mu_{A_{1}} \circ \mu_{A_{2}}\right)^{-}(u, v) \wedge\left(\mu_{A_{1}} \circ \mu_{A_{2}}\right)^{-}\left(u, v_{2}\right) . \\
=\min \left\{\mu_{A_{1}}^{+}(u) \wedge \mu_{A_{2}}^{+}(v), \mu_{A_{2}}^{+}\left(v_{2}\right)\right\}=\mu_{A_{1}}^{+}(u) \wedge \mu_{A_{2}}^{+}(v) \wedge \mu_{A_{1}}^{+}(u) \wedge \mu_{A_{2}^{+}}\left(v_{2}\right) \\
=\left(\mu_{A_{1}}^{+} \circ \mu_{A_{2}^{+}}\right)^{+}(u, v) \wedge\left(\mu_{A_{1}}^{+} \circ \mu_{A_{2}^{+}}\right)^{+}\left(u, v_{2}\right) \\
=\left(\mu_{A_{1}} \circ \mu_{A_{2}}\right)^{+}(u, v) \wedge\left(\mu_{A_{1}} \circ \mu_{A_{2}}\right)^{+}\left(u, v_{2}\right) .
\end{gathered}
$$

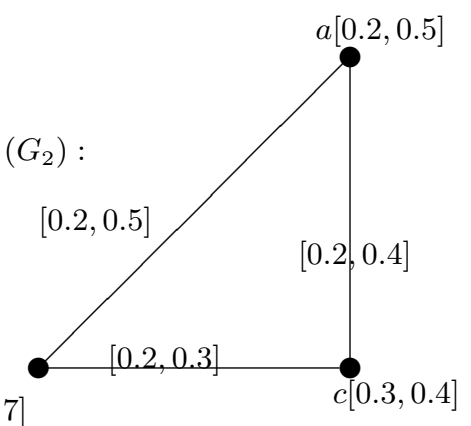

Fig $3.4 a$

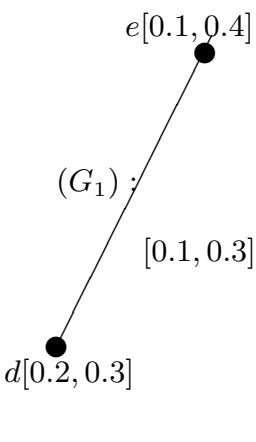

Fig 3.4b.

Case 3: $u \notin S_{1}$ and $v \notin S_{2}$. Since $S_{1}$ and $S_{2}$ are minimal dominating sets of $G_{1}$ and $G_{2}$, respectively. Therefore there exist $u_{1} \in S_{1}$ and $v_{1} \in S_{2}$, such that

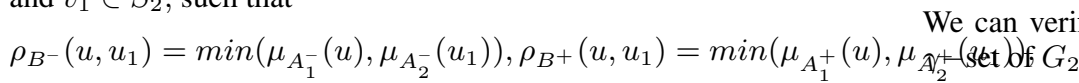

We can verify that $S_{1}=\{a\}$ is $\gamma-$ set of $G_{1}$ and $S_{2}=\{d\}$ is 
[14] L. A. Zadeh, "Fuzzy Sets", Information and Control, 8, 1965, 338-353.

[15] L. A. Zadeh, The concept of a linguistic and application to approximate reasoning I, Inform.Sci. 8 (1975) 149?249.

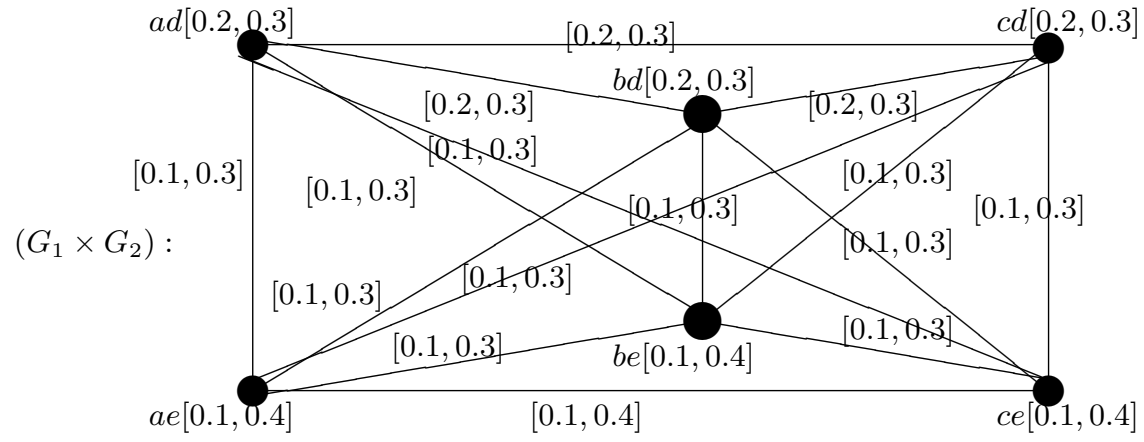

Fig $3.4 c: G_{1} \circ G_{2}$

We can verify that $S_{1} \times S_{2}=\{a d\}$ is a $\gamma-$ set of $G_{1} \circ G_{2}$ Hence $\gamma G_{1} \circ G_{2}=\left|S_{1} \times S_{2}\right|=|a d|=0.55$

\section{REFERENCES}

[1] M. Akram, Interval-valued fuzzy line graphs, Neural Computing and Applications, 21(1), (2012), 256-267.

[2] M. Akram and W. A. Dudek, Interval-valued fuzzy graphs, Compute. Math. Appl. 61(2), (2011), 289-299.

[3] P. Debnath, "Domination in interval-valued fuzzy graphs", Annals of Fuzzy Mathematics and Informatics, 6(2), (2013), 363-370.

[4] F. Haray, "Graph theory", Addison Wesley, Third printing, October, (1972).

[5] A. Kaufman, Introduction a La Theorie des Sous-ensembles Flous, Paris, Masson et cie Editeurs, (1973).

[6] J. N. Mordeson and C. S. Peng, Operations on fuzzy graphs, Inform. Sci. 79 (1994) 159?170.

[7] M. Naga Maruthi Kumari and R. Chandrasekhar, "Operations on interval-valued fuzzy graphs", IJARSE; 4(4),(2015), 618627.

[8] T. Pramanik, Sovan Samanta and Mahdumangai Pal, "interval-valued fuzzy graphs", International Journal of Fuzzy logic and Intelligent Systems, 20 (4), (2020), 316-323.

[9] A. Rosenfeld, Fuzzy graphs, In: L. A. Zadeh, K. S. Fu and M. Shimura, (Eds). "Fuzzy Sets and their Applications", Academic Press, 1975, New York.

[10] H. Rashmanlou, Sovan Samanta and M. Pal, "Some results on interval-valued fuzzy graphs", International Journal of Computer Science and Electronics Engineering , 3(3), (2015), 205211.

[11] A. Somasundaram, S. Somasundaran, "Domination in fuzzy graphs-I", Pattern Recognition Letters, 19, (1998), 787-791.

[12] A. Somasundaram, "Domination in fuzzy graphs-II", Journal of Fuzzy Mathematics, 20, (2000), 281-289.

[13] D.Umamageswari and P.Thangaraj, "Domination operation on bipolar fuzzy graphs", International Journal of Computational Research and Development, 2,(2017), 56-65. 